\title{
Assessment of Medication Adherence in Elderly Patients With Cardiovascular Diseases Based on Demographic Factors in Bushehr City in the Year 2013
}

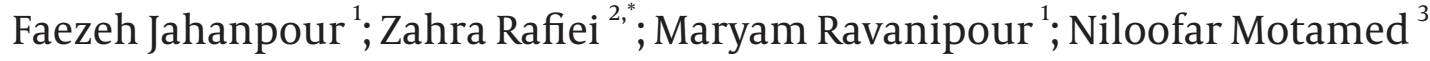 \\ ${ }^{1}$ Department of Nursing, Bushehr University of Medical Sciences, Bushehr, IR Iran \\ ${ }_{3}^{2}$ Student Research Committee, Department of Nursing, Bushehr University of Medical Sciences, Bushehr, IR Iran \\ ${ }^{3}$ Department of Social Medicine, Bushehr University of Medical Sciences, Bushehr, IR Iran \\ ${ }^{*}$ Corresponding author: Zahra Rafiei, Student Research Committee, Department of Nursing, Bushehr University of Medical Sciences, Bushehr, IR Iran. Tel: +98-9398373034, \\ E-mail:zahra_rafiee67@yahoo.com
}

Received: February 3, 2015; Accepted: March 6, 2015

\begin{abstract}
Background: The growing global elderly population is in need of more prescription medications.
Objectives: The present study assessed medication adherence with respect to demographic factors among elderly patients with cardiovascular disease who were admitted to a teaching hospital as well as outpatients referred to treatment centers in Bushehr, Iran.

Patients and Methods: The present study was a descriptive-analytic cross-sectional study using a simple random sampling method that investigated 125 elderly patients with cardiovascular disease. Data were collected through the Medication Adherence Questionnaire. Data were analyzed by using descriptive statistics, and Pearson and Cramer's V correlation analyses, using SPSS version 18.

Results: Among the patients, $57.6 \%$ and $42.4 \%$ were female and male, respectively. Moreover, $62.4 \%$ had high adherence to their prescribed medications. Frequency of medication use, history of previous hospitalization, and sex were significantly associated with adherence to medication $(\mathrm{P}<0.05)$.

Conclusions: More than half of elderly patients with cardiovascular disease had high adherence to their medications. Demographic factors including the frequency of daily medication use, history of hospitalization, and female sex were associated with adherence to medication. By considering these factors, doctors and nurses increase medication adherence, especially for older adults.
\end{abstract}

Keywords: Medication Adherence; Cardiovascular Diseases; Aged

\section{Background}

Aging is the final period of all organisms' life span and is more relevant than ever. However, as physical and mental aging vary among individuals, it is impossible to determine a specific cut-off for being old or specify exactly when a person can be considered elderly. Nevertheless, aging is a chronological process that generally starts at 65 years (1). Aging is a phenomenon that generally initiates as changes in the biology, physiology, biochemistry, and anatomy of the body's cells; these changes affect the performance of cells over time (2). The physical and mental changes caused by aging include gastrointestinal disorders (i.e. poor digestion/constipation), genitourinary problems (i.e. urinary incontinence), loss of teeth, bone problems (i.e. back and knee pain), osteoporosis, eye and ear diseases (i.e. loss of vision, hearing, etc.), cardiovascular diseases, eating disorders, psychiatric disorders (i.e. depression, anxiety, sleep disorders, dementia, poor memory, etc.), and inactivity (3).

The global elderly population is expected to reach to two billion in 2050. The elderly population in Iran is predicted to nearly triple in the 20 years spanning 2006 - 2026. Therefore, the country will face the social phenomenon of aging in the future decades (4). Considering the unique characteristics of the elderly period, many aged people frequently experience hospitalization for various reasons; the main causes are cardiovascular diseases such as hypertension, coronary artery diseases, and valvular heart diseases $(5,6)$. Cardiovascular diseases, the most common diseases in aging people, are progressive diseases that begin in childhood; however, their clinical manifestations mainly occur in adulthood and beyond. The rapid economic and social changes in recent decades in many Eastern Mediterranean and Middle Eastern countries including Iran have resulted in cardiovascular diseases being a major health and social problem. These diseases are the leading cause of death worldwide (one out of every three deaths), killing approximately 17 million people annually. If no special preventive action is taken by 2020 , this will increase to 24.8 million deaths annually (7).

Copyright (C) 2015, Ahvaz Jundishapur University of Medical Sciences. This is an open-access article distributed under the terms of the Creative Commons Attribution-NonCommercial 4.0 International License (http://creativecommons.org/licenses/by-nc/4.0/) which permits copy and redistribute the material just in noncommercial usages, provided the original work is properly cited. 
The appropriate use of prescription medications is an effective way to treat both acute and chronic diseases. Unfortunately, medications are not used as regularly as they are prescribed. One of the factors affecting the incidence of cardiovascular diseases is non-adherence (or non-compliance) to prescribed medications, which has been identified as a clinical problem with serious economic, social, and health-related consequences (8). The World Health Organization (WHO) defines adherence (or compliance) as the extent to which a patient follows drug use behaviors, suggested diet, or lifestyle changes in accordance with the healthcare providers' recommendations (9).

Non-adherence to medications causes approximately 125000 deaths in the United States annually; $10 \%$ of these fatalities occur in hospitalized patients and $23 \%$ occur in people receiving in-home hospice care (10). Only half of patients adhere to their medications (11). However, 47 - 100\% of elderly people are reported to adhere to medications (9).

Studies in this area emphasize that when the aging process begins, the prevalence of chronic diseases generally associated with pain, disability, and reduced quality of life incur the increasing need for medication use (12). Accordingly, a 75-year-old person is normally affected by 3.5 chronic diseases and is in need of using several medicines (13). Despite extensive research about adherence/ non-adherence to medications in the past five decades, little progress has been made towards solving this health problem; they report that 30 - 50\% of medications are not used as prescribed, which leads to decreased effectiveness and increased side effects and costs (14). The economic costs associated with non-adherence to medications is estimated to be 400 billion dollars worldwide (15).

Cardiovascular diseases are also considered among the first and most prevalent causes of death in Iran, resulting in high treatment costs. For example, in 1999, approximately 500 million dollars were spent on the treatment of cardiovascular disease patients (16). However, despite the pervasiveness of cardiovascular diseases, relatively little research has been conducted on medication adherence. Among the few studies in this area, Parsa-Yekta et al. examined 150 patients with coronary heart disease and found that only $28 \%$ used their medications appropriately (17).

Because of the importance of this problem, additional studies are urgently required to understand the severity of the problem and its related factors.

\section{Objectives}

The present study assessed the demographic variables related to medication adherence in elderly patients with cardiovascular diseases admitted to a teaching hospital as well as outpatients referred to treatment centers in Bushehr, Iran in 2013.

\section{Patients and Methods}

The present study was a descriptive-analytic cross-sectional study. The participants were elderly patients with cardiovascular diseases admitted to a teaching hospital as well as outpatients referred to treatment centers in Bushehr. The patients were selected by a simple random sampling method. To assure the patients' medical diagnoses, the medical records and self-reports of inpatients and outpatients, respectively, were used. The inclusion criteria were age over 60 years, a history of cardiovascular disease for more than 6 months, and a lack of cognitive or awareness problems. Patients with any other diseases such as diabetes, asthma, kidney failure, etc. were excluded.

Data about adherence to medications were collected through the Medication Adherence Questionnaire by Morisky; the reliability and validity (Cronbach $\alpha=0.67$ ) of the questionnaire have already been demonstrated in other Iranian investigations.

Morisky's questionnaire consists of four questions with five possible responses for each: 0 , always; 1 , often; 2 , sometimes; 3 , rarely; or 4, never. The sum of the points is used as an indicator of patients' adherence to their medications. Patients with scores $\geq 14$ and $<14$ are considered to have high and low adherence to medications, respectively.

The Cronbach $\alpha$ coefficient in the present study was 0.886 , confirming the reliability of the questionnaire. Moreover, information including age, sex, income, education level, history of hospitalization, number of medicines, daily medicine use frequency, and disease duration was collected through demographic questionnaires. All patients completed the questionnaires and were interviewed individually.

The Kolmogorov-Smirnov test was used to test the normality of the distribution of the data. The data were analyzed by using descriptive statistics (i.e. frequency, frequency percentage, mean, and standard deviation). Pearson's correlation coefficients were calculated to investigate the relationship between age and adherence, and $\chi^{2}$ and Cramer's $V$ coefficients were calculated to examine other demographic variables and adherence. The level of significance was set at $(\mathrm{P}<0.05)$.

Assuming an $\alpha$ of $5 \%$, test power of $90 \%$, and correlation of 0.3 between the patients' beliefs and their medication adherence, a required sample size of 112 was calculated by the following formula using Gpower software (Equation 1). Assuming a 10\% loss, 125 patients were required for the analysis. The collected data were analyzed by using SPSS version 18 .

$$
n=\frac{\left(\mathrm{z}_{1-\frac{\alpha}{2}}+\mathrm{z}_{\beta}\right)^{2}}{\frac{1}{4}\left[\operatorname{In}\left(\frac{1+p}{1-p}\right)\right]^{2}}+3
$$

\section{Results}

A total of 125 patients with cardiovascular diseases in Bushehr were analyzed; $57.6 \%(\mathrm{~N}=72)$ were female, and $42.4 \%(\mathrm{~N}=53)$ were male. The patients were 63 - 89 years old, with an average age of $74 \pm 6.4$ years. 
Concerning medication adherence, 37.6\% $(\mathrm{N}=47)$ and $62.4 \%(\mathrm{~N}=78)$ had low and high adherence to medications, respectively. Age was not significantly correlated with medication adherence $(R=0.009$, P value $=0.92)$. The relationships between medication adherence and demographic variables are presented in Table 1. Medication adherence was significantly associated with the frequency of medication use, history of hospitalization, and sex. The strongest relationship was observed between sex and medication adherence (Cramer's $V=0.63$ ).

\section{Discussion}

In the present study, $62.4 \%$ and $37.6 \%$ of the patients had high and low medication adherence, respectively, which is consistent with the study of Abbasi et al. (18) in which $60 \%$ of the patients with hypertension had high adherence to medications. In contrast, Parsa-Yekta et al. (17) report $56 \%$ of patients who suffer from heart diseases do not properly follow their medication use instructions. Similarly, in the other cohort study, only one-third of pa- tients showed adherence to medications (15). Self-reports were one of the tools used to collect data in the present study; therefore, one reason for this lack of consistency among studies might be due to patients reporting better adherence to their medications than reality. Another possible reason is the cross-sectional design of the present study. Among the demographic variables analyzed, sex, history of hospitalization, and frequency of medication use were significantly associated with medication adherence. Accordingly, $88.9 \%$ of female patients had high adherence to medications compared to only $26.4 \%$ of male patients. This finding is concordant with that of Emilsson et al. (19), who report that male patients with asthma have poorer adherence to medications than female patients. However, the present finding is inconsistent with that of Minaiyan et al. (20), who report no significant relationship between sex and medication adherence. This discrepancy among studies confirms that patients' adherence to medications can be influenced by their disease as well as demographic factors.

Table 1. Relationships between demographic variables and adherence to medications ${ }^{\text {a }}$

\begin{tabular}{|c|c|c|c|c|}
\hline Variable & High Adherence & Low Adherence & P-value & Phi or Cramer's V \\
\hline Gender & & & $0.0001^{b}$ & 0.63 \\
\hline Female & $64(88.9)$ & $8(11.1)$ & & \\
\hline Male & $14(26.4)$ & $39(73.6)$ & & \\
\hline History of hospitalization & & & $0.001^{b}$ & 0.30 \\
\hline Yes & $60(73.2)$ & $22(26.8)$ & & \\
\hline No & $18(41.9)$ & $25(58.1)$ & & \\
\hline Income & & & 0.23 & 0.15 \\
\hline$<500$ USD & $30(68.2)$ & $14(31.8)$ & & \\
\hline $500-1000$ USD & $42(62.7)$ & $25(37.3)$ & & \\
\hline$>1000$ USD & $6(42.9)$ & $8(57.1)$ & & \\
\hline Number of daily medicines & & & 0.07 & 0.20 \\
\hline$<3$ & $3(42.9)$ & $4(57.1)$ & & \\
\hline $3-5$ & $54(58.7)$ & $38(41.3)$ & & \\
\hline$>5$ & $21(80.8)$ & $5(19.2)$ & & \\
\hline Frequency of medicine use & & & $0.001^{b}$ & 0.30 \\
\hline 1 or 2 & $34(49.3)$ & $35(50.7)$ & & \\
\hline 3 & $44(78.6)$ & $12(21.4)$ & & \\
\hline Disease duration, y & & & 0.17 & 0.16 \\
\hline$<5$ & $44(56.4)$ & $34(43.6)$ & & \\
\hline $5-9$ & $20(69)$ & $9(31)$ & & \\
\hline$>9$ & $14(77.8)$ & $4(22.2)$ & & \\
\hline Education level & & & 0.79 & 0.06 \\
\hline Illiterate & $59(63.4)$ & $34(36.6)$ & & \\
\hline Elementary & $12(63.2)$ & $7(36.8)$ & & \\
\hline Above elementary & $7(53.8)$ & $6(46.2)$ & & \\
\hline
\end{tabular}

a Values are presented as No. (\%).

$\mathrm{b}$ Indicates the significance of the relationship. 
Regarding the relationship between history of hospitalization and medication adherence, patients with a history of hospitalization showed better adherence to their medications, which does not support the hypothesis discussed in the review of van der Wal et al. (21), i.e. heart failure patients with a history of hospitalization have poorer adherence to medications than patients without any history of hospitalization; however, the authors also mention that this hypothesis is not generalizable to all patients. Nevertheless, more research is required for clarification because of the lack of investigations regarding the relationship between history of hospitalization and medication adherence. One possible explanation for the findings of the present study is that patients with a history of hospitalization, who may have experienced pain (especially severe angina pain), tolerated aggressive procedures, experienced hospitalization-related complications, etc., better adhere to their medications to prevent experiencing these issues again.

In the present study, increasing frequency of daily medication use was associated with better medication adherence. For example, patients who used medications three times per day (i.e. morning, noon, and night) showed better adherence. However, this finding contrasts with that of Iskedjian et al. (22), who report that patients who took medications once per day showed better adherence. Considering most elderly people live with their children or close relatives owing to physical limitations, this discrepancy between the present and previous studies might be due to greater attention paid by patients and their family members to the use of daily medications. Moreover, as medications are usually prescribed to be taken after meals, medications used three times per day (after each meal) are less likely to be ignored than those taken only once per day (e.g. at 09:00).

In the present study, no significant relationship was observed between medication adherence and other demographic variables such as age, income, and education level. Conversely, Gottlieb et al. (23) report a significant negative relationship between age and medication adherence, and Kennedy and Morgan (8) report a significant relationship between income and medication adherence.

This study has some limitations. First, because of a lack of available patients, the present study was conducted on both inpatients and outpatients; therefore, it is possible the hospitalized patients showed better adherence to their medications than reality because of their current health situation in the hospital as well as the attention paid by nurses and their families about their use of prescribed medications. Second, because of the limited sample size, it is difficult to generalize the findings of the present study; therefore, similar studies with larger sample sizes are strongly recommended. Moreover, analyzing the separated samples of inpatients and outpatients groups will be useful.

In conclusion, demographic factors including sex, frequency of daily medication use, and history of hospi- talization are associated with medication adherence in elderly patients with cardiovascular diseases. Given the importance and negative consequences of the issue of non-adherence to medication, it is necessary to consider factors influencing adherence/non-adherence to medication to improve patients' health and family situations. It is very important that doctors and nurses, who play significant roles in disease prevention and treatment, consider these factors. By considering the importance of these factors, it will be possible to promote patients' adherence to medications and consequently improve their lives.

\section{Acknowledgements}

We would like to thank all of the staff members who aided the preparation of this study as well as the dear elderly patients who helped us conduct this study.

\section{Authors' Contributions}

Study concept and design: Zahra Rafiei and Faezeh Jahanpour; data analysis and interpretation: Niloofar Motamed; manuscript preparation: Zahra Rafiei, Faezeh Jahanpour, and Maryam Ravanipour; data collection: Zahra Rafiei; critical revision: Faezeh Jahanpourr, Niloofar Motamed, and Maryam Ravanipour.

\section{Financial Disclosure}

The Deputy of Research of the Bushehr University of Medical Sciences provided financial support for this research.

\section{Funding/Support}

This study was supported by the Bushehr University of Medical Sciences, Bushehr, IR Iran.

\section{References}

1. Seyed Mirzaie M. [Some Observations on the Dimensions of Aging With a Look at Japan's Experience]. Human Sci J. 2007;53:195-216.

2. Ameri GF, Govari F, Nazari T, Rashidinejad M, Afsharzadeh P. [The adult age theories and definitions]. Hayat. 2002;8(1):4-13.

3. World Health organization.. [Active aging: apolicyframework].Mashhad: Mashhad university of medical science; 2003.

4. Qysryan I. [Socialdimensionsofeconomic,agingin Iran]. Il of Population. 2010. Available from: http://apps.who.int/iris/bitstream/10665/67215/3/WHO_NMH_NPH_02.8_jpn.pdf?ua=1.

5. Shojaei A. [The health costs and diseases in Medical Services Insurance Organization,Tehran province,1386]. Iranian Journal of Ageing. 2008;6(22).

6. Fakhrzadeh H, Sharifi F. [Cardiovascular diseases in the elderly].J of Gorgan Uni of Med Sci. 2012;14(3):1-9.

7. Sharifirad G, Mohebbi S, Matlabi M. [The relationship of physical activity in middle age and cardiovascular problems in old age in retired people in Isfahan, 2006]. The Horizon of Med Sci. 2007;13(2):57-63.

8. Kennedy J, Morgan S. A cross-national study of prescription nonadherence due to cost: data from the Joint Canada-United States Survey of Health. Clin Ther. 2006;28(8):1217-24.

9. Tsai KT, Chen JH, Wen CJ, Kuo HK, Lu IS, Chiu LS, et al. Medication adherence among geriatric outpatients prescribed multiple medications. Am J Geriatr Pharmacother. 2012;10(1):61-8. 
10. Peterson AM, Takiya L, Finley R. Meta-analysis of trials of interventions to improve medication adherence. Am J Health Syst Pharm. 2003;60(7):657-65.

11. McDonald HP, Garg AX, Haynes RB. Interventions to enhance patient adherence to medication prescriptions: scientific review. JAMA. 2002;288(22):2868-79.

12. Zareban I, Shamsi M, Kuhpayehzadeh J, Baradaran H. [The effect of education based on extended parallel process model (EPPM) on the self-medication of elderly in Zarandieh].J of Kermanshah Uni of Med Sci. 2013;17(8):501-8.

13. Yavari HR,, AkbariKamrani AA, Bakhshi E, Saboor M, Sahhaf R. [Association between Polypharmacy and Socio-demographic factors among elderly residents of Kahrizak Charity Foundation, Tehran, 2010-2011]. Iran J of Ageing. 2014;8(31).

14. Hekmatpou D, Mohammadi E, Ahmadi F, Arefi SH. [Non-compliance factors of congestive heart failure patients readmitted in cardiac care units]. Iran J of Critical Care Nursing. 2009;2(3):91-7.

15. Cannon CP. Cardiovascular disease and modifiable cardiometabolic risk factors. Clin Cornerstone. 2007;8(3):11-28.

16. Shamsi A, Ebadi A. [Risk Factors of Cardiovascular Diseases in Elderly People]. Iran J of Critical Care Nursing. 2011;3(4):187-92.

17. Parsa-Yekta Z, Zakeri Moghaddam M, Mehran A, Palizdar M. [Study of medication compliance of patients with coronary heart diseases and associated factors]. Hayat. 2003;9(4):34-43.

18. Abbasi M, Salemi S, Fatemi NS, Hosseini F. [hypertensivepatients, their compliance level and it's relation to their health beliefs]. Iran Jl of Nursing. 2005;18(41):1-2.

19. Emilsson M, Berndtsson I, Lotvall J, Millqvist E, Lundgren J, Johansson A, et al. The influence of personality traits and beliefs about medicines on adherence to asthma treatment. Prim Care RespirJ. 2011;20(2):141-7.

20. Minaiyan M, Taheri M, Mirmoghtadaee P, Marasi M. [Comparative Role of Demographic Factors and Patient's Belief about Prescribed Medicine on Adherence to Drug Treatment in Chronic Diseases].Journal of Isfahan Medical School. 2011;29(156):1469-77.

21. van der Wal MH, Jaarsma T, van Veldhuisen DJ. Non-compliance in patients with heart failure; how can we manage it? Eur J Heart Fail. 2005;7(1):5-17.

22. Iskedjian M, Einarson TR, MacKeigan LD, Shear N, Addis A, Mittmann N, et al. Relationship between daily dose frequency and adherence to antihypertensive pharmacotherapy: evidence from a meta-analysis. Clin Ther. 2002;24(2):302-16.

23. Gottlieb S, Behar S, Schwartz R, Harpaz D, Shotan A, Zahger D, et al. Age differences in the adherence to treatment guidelines and outcome in patients with ST-elevation myocardial infarction. Arch Gerontol Geriatr. 2011;52(1):118-24. 